To appear in: M. Slater and Z. Yudell (ed.), Metaphysics in the Philosophy of Science, Oxford University Press.

\title{
Time for empiricist metaphysics
}

\author{
Katherine Brading \\ University of Notre Dame \\ kbrading@nd.edu
}

\begin{abstract}
At the beginning of Newton's Principia there is a famous scholium where he discusses time, space, place and motion using three distinctions: absolute and relative; true and apparent; mathematical and common. I discuss these three distinctions for the case of time, arguing for their significance for the metaphysics of time, and for the claim that Newton's empirical methods reach deeply into metaphysical questions concerning the nature and structure of time. I suggest that this example provides important lessons for how philosophers should approach the relationship between metaphysics and empirical science.
\end{abstract}

\section{Introduction}

To what extent are the details of empirical enquiry relevant for the metaphysics of time? I shall argue that they are deeply, utterly and inextricably entwined, and moreover that they became so as a consequence of philosophical moves made by Newton in his Principia. Prior to the Principia, general questions about the nature and structure of time, such as whether or not time is merely an aspect of material change, whether there is one time or many, whether time is inherently metrical, and so forth, could be (and were) appropriately addressed via arguments based on broadly a priori considerations. In the wake of Newton's Principia, this is no longer the case. Newton showed how the answers to these questions depend on the intricate details of empirical enquiry. Those of us who are interested in the metaphysics of time are not free to pretend that the philosophical moves made by Newton were never, in fact, made. He made these moves, and this paper is about their implications for the metaphysics of time.

In the recent Blackwell Companion to Philosophy of Time (2013), there is a section on "The History of the Philosophy of Time", and there between a chapter on creation and eternity in medieval philosophy, and one on classical empiricist discussions of time, we find a chapter on Newton. In this 
chapter, Eric Schliesser asks us to pause with Newton, and to look in more detail at his contributions to the philosophy of time. Instead of taking Newton's physics, and then looking at what other philosophers have to say about time in the light of his physics, we look at what philosophical moves Newton himself made. I think Schliesser is right that this is worth doing, and I claim that Newton's empirical methods reach deeply into metaphysical questions concerning the nature and structure of time. For philosophers with an interest in the metaphysics of time, Newton's Principia needs to be read as a philosophical text, offering contributions to an empiricist metaphysics of time. ${ }^{1}$

I begin from three distinctions that Newton made at the beginning of his Principia, in the famous scholium on time, space, place and motion. There, he said that we should distinguish between absolute and relative, true and apparent, and mathematical and common, for each of time, space, place and motion. I outline these distinctions as they apply to time (section 2), and then discuss Schliesser's (2013) interpretation of Newton's distinction between absolute and true time (section 3), explaining why I think a different approach is needed. I then build towards the positive conclusions that I want to draw. I begin by offering an alternative interpretation (section 4), according to which Newton is drawing on existing terminology and implicit conceptual distinctions in order to make explicit and systematic a three-way set of distinctions concerning the nature and structure of time. In so doing, he makes a contribution to the philosophy of time. I then argue that (a) these distinctions are empirically accessible (see section 5), and (b) all three distinctions are necessary for setting up the project of the Principia (see section 6). It follows from this, I argue, that certain questions concerning the nature and structure of time become empirically tractable through the pursuit of that project, or some appropriately similar project. By situating Newton's Principia in the appropriate philosophical context (section 7), we can read the Principia as a direct contribution to the metaphysics of space, time, matter and motion, and as offering an empiricist metaphysics of a particular kind. I claim that Newton refined the conceptual distinctions appropriate for asking questions about the nature and structure of time, and transformed the methdology by which such questions should be addressed, and I show this in detail for each of the three distinctions he makes at the outset of the Principia (see section 8). I conclude by drawing together the results of the preceding sections, and then use the specific example tackled in this paper to suggest some more general lessons about how philosophers should approach the relationship between metaphysics and empirical science (section 9).

\footnotetext{
${ }^{1}$ I take the phrase "empiricist metaphysics" from Janiak (2004, p. 29) who, in describing the re-appraisal of Newton due to Stein, writes: "The result is not an anti-metaphysical Newton but a kind of empiricist metaphysician."
} 


\section{Three distinctions}

Newton's Principia (published in 1687) opens with a series of definitions of the terms that he will use, including "quantity of matter", "quantity of motion", "inherent force of matter", and so forth. Immediately following these definitions he turns his attention to time, space, place and motion, in a scholium which begins as follows (Cohen and Whitman, 1999, p. 408):

Although time, space, place and motion are very familiar to everyone, it must be noted that these quantities are popularly conceived solely with reference to the objects of sense perception. And this is the source of certain preconceptions; to eliminate them it is useful to distinguish these quantities into absolute and relative, true and apparent, mathematical and common.

The literature has largely focused on absolute versus relative motion, and absolute space, with comparatively little discussion of time, ${ }^{2}$ and nothing that I know of about why Newton has this threefold set of distinctions.

However, Schliesser's (2013) discussion of Newton on time was inpired by Huggett (2012), who offered an interpretation of the distinction between absolute and true motion. ${ }^{3}$ I differ from both in my interpretation of the terminology, and therefore in the distinctions that Newton is drawing. In this paper I focus on the case of time.

Newton introduces his discussion of time as follows:

Absolute, true, and mathematical time, in and of itself and of its own nature, without reference to anything external, flows uniformly and by another name is called duration. Relative, apparent, and common time is any sensible and external measure (precise or imprecise) of duration by means of motion; such a measure - for example, an hour, a day, a month, a year - is commonly used instead of true time.

\footnotetext{
${ }^{2}$ Arthur, 1995; Gorham, 2012; McGuire, 1978; Palmerino, 2013.

${ }^{3}$ The disagreements that I have with Schliesser and Huggett are small compared to the overall content of their papers, from which I learned much. As so often happens, the many points of agreement and enlightenment I pass over in silence, to focus on what we may learn from a point on which we disagree.
} 
Thus, Newton is explicit in applying to time the three distinctions of absolute versus relative, true versus apparent, and mathematical versus common. What does he mean by these distinctions, and why do they matter?

According to Schliesser (2013), the terminology of "absolute" and "true" marks a distinction between time as a theoretical construct, arrived at from empirical considerations and applied in the context of the project of the Principia (absolute time), versus time as it occurs in metaphysical and theological discussions (true time). He writes (p. 91):

True time is an unnecessary addition to Newton's conceptual framework of absolute and mathematical time given the particular problems addressed in the Principia.

And he goes on (p. 92):

Newton introduces more conceptual distinctions than are required by his physical theory; his dynamics requires no more than absolute (mathematical) time as a contrast to "relative, apparent, and common time" without resort to "true" time.

On Schliesser's view, while it plays an important role in Newton's overall philosophy of time, "true" time (and therefore the contrast between true and apparent time) has no place in the empirical project of the Principia.

I disagree. And what is at stake here is not merely Newton exegesis: resolving the terminological issue has implications for which questions concerning the nature and structure of time Newton's empirical project is capable of addressing. In other words, what is at stake is the metaphysical reach of his empirical methods. I think, contra Schliesser, that all three of Newton's conceptual distinctions between absolute and relative, true and apparent, and mathematical and common time, have empirical import (section 5) and are necessary for the project of the Principia (section 6). Moreover, I maintain that these distinctions bear on long-standing philosophical questions concerning the nature and structure of time (sections 7 and 8). As a consequence, these questions become subject to empirical investigation in a manner not possible prior to the relevant philosophical innovations of Newton's Principia.

In the following section I discuss Schliesser's approach in some more detail, before turning to my preferred approach in section 4, below.

\section{Schliesser's account of Newton on time}


Schliesser (2013) argues that we should distinguish absolute time from true time, and I think he is right that the two terms are not synonymous for Newton. However, whereas for Schliesser "absolute time" is a mathematical construct, a time parameter in our physical theory by which we seek to place a measure on the passage of true time, I will argue below that "absolute" and "true" are both to be understood as characteristics of time, characteristics that will both be represented in an appropriate time parameter of an adequate physical theory.

Schliesser's proposal is that absolute time is a theoretical construct obtained from our observations of the heavenly bodies and their relative motions via the equation of time (of which more below). He writes (2013, p. 90):

As a first approximation, we can say that "absolute" time is approximated by our clocks (or some other measure of relative time) corrected by the astronomical equation of time ... allowing thus a measure of true time.

On this view, the empirical basis of "absolute time" is such that it extends spatially only so far as the bodies in our planetary systems whose relative motions we observe (with respect to the background of the fixed stars). True time, by contrast, is that which we are seeking to approximate in our construction of absolute time and, according to Schliesser, it extends from infinity to infinity. Moreover, insofar as there is always room for improvement in the process by which we construct absolute time, we can think of it as a "regulative ideal".

Schliesser's "absolute time" is needed for the project of the Principia because what Newton is trying to do in the Principia is to determine whether our planetary system is geocentric or heliocentric, and in order to solve this problem he needs to be able to assign accelerations to bodies in our planetary system in a unique and consistent way. This latter task requires a single time parameter relative to which the bodies in our planetary system are assigned their motions. Furthermore, for the purposes of the project, the spatial extent of this time parameter need be only so far as the observed bodies in our planetary system, and this is consistent with the empirical basis from which the time parameter is constructed.

For Schliesser, our empirically constructed (absolute) time is only approximately accurate compared to metaphysical (true) time, and it has limited spatial extent. When Newton introduces true time, in addition to absolute time, he does not thereby introduce any new empirical questions, because true time is answerable to the demands of Newton's rational theology and not to empirical 
considerations. The only open empirical question concerns the spatial extent of absolute time: will the time parameter appropriate for our planetary system turn out to be appropriate for treating bodies outside our planetary system?

This is one way to read the distinctions, and to think about their significance for philosophy of time. I agree up to a point. I agree that Newtonian absolute time should not be conflated with Newtonian true time. I agree that for the purposes of the Principia Newton does not need his time parameter to extend from infinity to infinity. I agree that the spatial reach of Newton's time parameter in the Principia is an empirical matter. However, I do not think that for Newton "absolute" and "true" mark Schliesser's distinction between a spatially limited empirical time parameter and a theologically motivated infinitely extended "time".

Initial doubts about Schliesser's interpretation arise when we notice that in the scholium Newton does not make the positive assertion that absolute, true or mathematical time are eternal in duration, nor does he assert that space is infinite, and nor does he assert that each moment of time extends from infinity to infinity. We are familiar with these claims from other places in Newton's writings, but in this part of the text, where Newton is setting out what is needed for the project of the Principia, no such positive claims are made.

Moreover, the distinction that Schliesser draws is not one that we find doing work for Newton in his argument in the Principia, such that he has reason to mark it by means of a terminological distinction. As evidence for this, consider that Newton has just as good reasons to think that his absolute time extends to the physics of the distant stars and to the planetary systems around distant stars (if any such exist) as he does to think that his laws of motion and law of universal gravitation apply to such bodies, and he does not make the solar system the boundary of applicability for these latter. Newton worried about how we extend our knowledge to bodies beyond the reach of our experiments, and this worry is explicity addressed in his Rule 3 of Reasoning, added to Book 3 in the second edition of the Principia (Cohen and Whitman, p. 795):

Those qualities of bodies that cannot be intended and remitted and that belong to all bodies on which experiments can be made should be taken as qualities of all bodies universally.

This rule plays a crucial role in enabling Newton to extend results from terrestrial experiments to the celestial bodies of the solar system. In applying Rule 3 to bodies beyond the solar system, we would certainly be wise to be tentative given the flimsiness (even non-existence) of our empirical evidence, but 
there is nothing in Newton's writings to indicate a sharp cut-off at the outer edges of the solar system such that we should not consider distant stars to be bodies. On the contrary, the possibility of other worlds around other Suns, governed by the same laws, is very much part of Newton's thinking. For example, there is a manuscript ${ }^{4}$ in which Newton asserts that the fixed stars are bodies just like our Sun: they are formed into spheres by their own gravity, and since they are bodies they are, by definition, subject to the laws of motion. It seems to me that the distinction Schliesser draws is not important for Newton's purposes.

Finally, the contexts in which Newton extends moments of time to spatial infinity are generally also those in which he is talking about God's presence in the world, rather than those in which he is concerned with methods of reasoning from the phenomena.

In my opinion, we have good reason to suspect that Newton was careful not to over-reach empirically when he was setting out his accounts of time and space in the scholium (i.e. at the outset of the empirical project of the Principia). There is therefore reason to doubt that the inclusion of "true time" is an "unnecessary addition" (Schliesser, 2013, p. 91). In the following section I propose an alternative interpretation of the terminology, in which each of his three distinctions - absolute versus relative, true versus apparent, and mathematical versus common - are relevant and important for the project of the Principia.

\section{An alternative interpretation}

When it comes to motion, there is a standard way of understanding the absolute versus relative terminology: relative motion is motion of a body with respect to other bodies, and absolute motion is motion of a body independent of its relations to other bodies (but perhaps in relation to absolute space and time instead). I take this to be uncontroversial. The idea of motion as relative to other bodies was familiar (from Descartes, among others), and much discussed, and moreover this is the one distinction that Newton himself explains in detail. He uses it in the same way for space, time, and place as he does for motion, and absolute motion is further spelled out in terms of absolute space and absolute time. Newton is clear about this terminology, and I take it that his readers would have understood his use of the terms in the way that is now standard. Absolute time is independent of material bodies, whereas relative time is an aspect of material bodies or of the relations among them.

\footnotetext{
${ }^{4}$ See Hall and Hall, 1962, pp. 374-7.
} 
The terms "true" and "apparent" have meanings which would already have been familiar to Newton's readers from the dispute over the Copernican system, so some historical context is useful. The Copernican dispute concerns whether the system of the world is geocentric or heliocentric or whether there is no fact of the matter. Book III of Newton's Principia is called "The System of the World" and this is where Newton marshals the resources developed in Books I and II to give his answer to the Copernican question. Addressing this question is the overall purpose of the Principia, and the Copernican dispute is therefore the appropriate context for understanding what Newton means by the terminology "true and apparent" in the Principia.

Within this dispute, those in the geocentric and heliocentric camps shared a commitment to true motion, as distinct from apparent motion: whatever the apparent motion of a given body (e.g. the Sun moving across the sky), and of which there may be many (depending on the position of the observer), there is nevertheless a unique motion that is the true motion of that body. Thus, for geocentrists and heliocentrists alike, one motion is singled out as not mere appearance, but proper to the body, and this is its true motion. The distinction between true and apparent motion comes to the fore in the Copernican dispute because of the obvious conflict in Copernicus's system between the appearances (it appears to us, observing from Earth, that the Sun moves around a stationary Earth) and the true motions described in the theoretical system (the Earth in motion around a stationary Sun), but even those astronomers committed to a geocentric theory made the distinction between the apparent motions of the heavenly bodies (how their motions appear to us) and their true motions.

With these distinctions in hand (between absolute versus relative and true versus apparent) there remains the issue of the relationship between them. For example, Descartes sought to give a relative account of true motion, whereas Newton sought to define true motion in terms of absolute motion. In his Principles II.25, Descartes defined true motion in terms of the relative motion of a body with respect to the immediately surrounding bodies (themselves considered to be at rest), and thereby sought to identify the one true relative motion proper to the body (i.e. its true motion) from the many relative motions that this body in the plenum undergoes. One of the things that Newton tried to do in the scholium to the definitions in the Principia was to show that true motion should be identified not with relative motion but with absolute motion, where for Newton absolute motion is motion with respect to absolute space and absolute time.

So these distinctions, and also I think the terminology, were already out there for the case of motion. Newton gathers them together, sets them out as two distinct pairs, and standardizes them 
across space, time, place and motion. If this is the right way to approach the terminology, ${ }^{5}$ then the contrast between true and apparent time is the following. Just as true motion is unique and proper to the body (or system of bodies) in question, in contrast to being a property of the appearances, so too true time is unique and proper to the body (or system of bodies) in question, a property of the body or system itself rather than of the appearances. We can illustrate the idea as follows. The relative motions of the Sun and Moon with respect to the Earth give rise to their apparent motions with respect to the fixed stars, as viewed from Earth. Each apparent motion may each be used as a clock for the Earth-SunMoon system, and therefore each gives rise to an apparent time (apparent solar time and apparent lunar time respectively). But these "clocks" do not tick regularly with respect to one another, and therefore the true time of the Earth-Sun-Moon system (if any such exists) remains to be determined.

The terminology of "mathematical" and "common" has its origins not in discussions of motion, but in the treatment of time in mathematical astronomy. In the scholium, Newton writes that absolute time is distinguished from relative time by the equation of common time. We can therefore begin our investigation of what is meant by "common time", and by the contrasting term "mathematical time", by looking at the equation of common time. Common time, in this context, is time on which a metric is imposed by means of material clocks, for the purposes of our common life; it is the division of the passage of time into intervals by which the rhythm of our lives is marked out, and has no more precision, nor any other properties, than those needed for this purpose. ${ }^{6}$ In the seventeenth century, apparent solar time (of which more below, see section 5) was used as the basis of common time. By contrast, the equation of common time was used by astronomers to construct a time parameter suitable for the purposes of mathematical astronomy (as explained below, section 5). This time parameter has precise mathematical properties (it is metrical, it is continuous, and so forth), and in later sections of this paper I will stress the importance of Newton's time parameter being metrical. This time parameter was of no interest or use to anyone except mathematical astronomers. For Newton's intended readership, well-versed in the problems of horology and mathematical astronomy (especially Huygens), this terminology of mathematical and common time would have been readily understood.

I claim, therefore, that all six terms in the three distinctions have meanings that are prior to, and external to, the project of the Principia. What's new with Newton is stating them all explicitly as

\footnotetext{
${ }^{5}$ See Huggett (2012) for an alternative.

"It would be interesting to know whether there is any connection between this use of the term "common time", and that in music (current at the time) where 4/4 time was considered "imperfect" and was known as "common time", whereas time signatures with a three-measure (e.g. 3/4 time) were considered "perfect" (in accordance with the trinity).
} 
contrasts like this, stating them all together, and applying them uniformly and systematically across time, space, place and motion.

If this is right, then all three distinctions are associated with independent questions concerning the nature and structure of time. Is time absolute or relative? Is time true or apparent? Is time mathematical or common? Moreover, as I will argue in what follows, each distinction has empirical import (section 5), all three conceptual distinctions are needed for setting up the project of the Principia (section 6), and therefore each of the three questions becomes subject to empirical investigation (section 6). The upshot is that, at the very least, there are more open empirical questions concerning time than Schliesser's interpretation of this terminology would allow. But there is much more than this. In making these distinctions explicit for the first time, and in tying them to the details of empirical enquiry, Newton makes the questions about the nature and structure of time more fine-grained, and transforms the process by which we are to address them. I will argue (section 8) that all three distinctions engage with familiar metaphysical questions concerning the nature and structure of time, and so those questions themselves become empirically tractable in Newton's hands. His work in the Principia thus constitutes an important transformation in the appropriate methodology for pursuing the metaphysics of time.

\section{Empirical access}

With the distinctions between absolute and relative, true and apparent, and mathematical and common understood as I suggest, I will now argue that they are empirically accessible, and therefore subject to empirical investigation. Moreover, this is something of which Newton was well aware at the time he was writing the Principia.

Our empirical access to time is indirect, via material bodies: we make use of material systems which exhibit periodic phenomena, i.e. which tick. The most obvious periodic phenomenon in our daily lives is the cycle of day and night itself. Traditionally, and into the fifteenth century, the hour was defined by taking the time from sunrise to sunset and dividing it into twelve (and similarly for the twelve hours of the night). ${ }^{7}$ Call this seasonal time. Time understood in this way is relative (it depends on the relative motion of the Sun and the Earth) and apparent (deriving from the motion of the Sun as it appears to us).

\footnotetext{
${ }^{7}$ For a detailed discussion of the historical and conceptual development of time measurement, see Audoin and Guinot, 2001.
} 
How would the division of day and night each into twelve hours be achieved? During the day, the Sun appears to us to move steadily across the sky, so the apparent motion of the Sun (assumed to be constant) could be used to divide the day into twelve. During the night, the stars similarly appear to move steadily across the sky, so the apparent motion of the stars (assumed to be constant) could be used to divide the night into twelve. One upshot of this approach seems to be that daytime hours in summer are longer than daytime hours in winter. But how can that be? We defined the hour as our unit of time, and by definition each hour must be of the same duration: an hour. So what does it mean to say that hours vary in duration, and how could we show that this is the case? One way to tell is to compare these seasonal hours with other periodic phenomena, such as the number of times sand runs through an old-fashioned egg-timer (or an hourglass!) in the course of an "hour"; even our own biological rhythms are sufficient for us to be able to "experience" a difference in length of seasonal hours.

In other words, there are different apparent times, arising from different choices of bodies whose relative motions we use to construct a clock. Faced with such disagreement, it can be convenient for a society to make a choice that is shared among its members. We adopt a common time, and in the mid seventeenth century apparent solar time was used for this purpose. Apparent solar time is defined as follows: take one complete circuit of the Sun around the Earth and divide it equally into 24 hours, so that one hour corresponds to the Sun moving through an angle of $15^{\circ}$.

Apparent solar time is a relative conception of time (depending on the relative motion of the Sun and the Earth). During the day, it is directly observable (look and see how long it takes for the Sun to move through $15^{\circ}$, or measure this indirectly by means of a sundial). During the night, we need theory to calculate how far the stars have to move for a corresponding $15^{\circ}$ motion of the Sun (postulated to be continuing its motion around the other side of the Earth). With this calculation in hand, such "apparent solar time" is observable - it is in the appearances and is therefore apparent.

When compared to other periodic phenomena, this is a great improvement: those phenomena are now in much greater agreement with our chosen clock. So there is good reason for ordinary people to switch from seasonal time to apparent solar time as the basis for common time, and many cities and towns did so. According to Audoin and Guinot (2001, p. 40), apparent solar time was in use as the standard for common time in country areas of Europe until the beginning of the twentieth century.

Astronomers too would have had good reason to prefer apparent solar time over seasonal time as their time parameter for astronomy. They were engaged in the task of predicting the motions of the heavenly bodies (the stars, the Sun, the Moon and the planets) using uniform circular motion. Any deviations of these bodies from uniform circular motion needed to be accounted for theoretically. By 
adopting apparent solar time rather than seasonal time, the motions of the heavenly bodies become less irregular. That is to say, they are less irregular with respect to equal intervals of time defined using apparent solar time. The role of theory in our understanding of time is clear: if one choice of clock (the standard of time that we adopt) yields the result that the motions are highly irregular, while another choice of clock yields the result that the motions are close to regular, then the second clock makes our theoretical task easier as we try to account for remaining irregularities.

Despite its great practical utility as a basis for common time, apparent solar time was nevertheless deemed "irregular" even by ancient astronomers. They worked instead with mean solar time, which is constructed as follows. Every day, when the Sun rises, it rises at a slightly different point relative to the background of the stars. A solar year is the time taken for the Sun to rise again at that same point plotted against the background of the stars. In the course of this year, if we plot the position of the Sun at sunrise every day with respect to the stars, we see that the Sun makes a complete journey around the sky, along a path called the ecliptic. This path is so important that we keep track of it at night (when we can't see the Sun) by means of the constellations of the zodiac. But the Sun speeds up and slows down during the year (passing through a greater or lesser angular distance along the ecliptic on different days). If we smooth out the motion of the Sun, so that it moves at constant daily speed around the ecliptic, the resulting position with respect to the fixed stars is the position of the mean Sun. ${ }^{8}$ Except when the actual Sun and the mean Sun coincide, no material body is located at the position of the mean Sun. But, if we use the mean Sun as our clock, the motions of the heavenly bodies overall become more regular: regularities in the irregularities are removed. This serves our goal of constructing a predictively adequate theory using regular motions: irregularities in the appearances (the apparent motions) are to be accounted for by such things as the eccentricity of the Sun's orbit, and so forth. Mean solar time "corrects" apparent solar time by removing periodic irregularities via the "equation of common time". 9 Notice that we are using theory here to move from apparent time, derived from the most regular

\footnotetext{
${ }^{8}$ Notice that this attributes all the irregularities to the Sun. The motion of the stars with respect to the Earth is assumed to be uniform, and this is used as the regular background with respect to which the apparent motion of the Sun is then smoothed out to construct the mean solar time. The uniform motion of the stars is equivalent to assuming that the daily rotation of the Earth is uniform. According to Audoin and Guinot (2001, pp. 46-8), Kepler mentioned the possibility of some irregularities in the Earth's rotation, Flamsteed was the first (1677) to try to detect them (without success), Maupertuis (1752) wondered whether there might be some irregularities and if so what their cause could be, and Kant suggested that "there could be slowing down effect due to dissipation of energy in tidal movements of the oceans. He was right, but the idea was only confirmed by observation two centuries later". Laplace (1825) rejected the possibility out of hand. It was not until the late nineteenth century that studies of the moon provided evidence of tiny irregularities in the Earth's rotation, with confirmation being due to Brown and de Sitter (1927), building on the work of Hansen and Newcomb.

${ }^{9}$ For more details, see Audoin and Guinot, 2001, pp. $40 \mathrm{ff}$.
} 
apparent motions that we observe (celestial motions), to an abstract theoretical time. Mean solar time is a theoretical construct: no material bodies used in its construction were observed to move regularly with respect to mean solar time, and it was therefore neither apparent nor relative. Astronomers knew of no relative motions that could serve as a clock with respect to this time. Thus, the "time" that is appropriate for astronomy is mathematical, and it is neither relative nor apparent. This is a conclusion that we arrive at through an inter-play between theoretical and empirical considerations, in which a theory of planetary motions is constructed by assigning simple basic motions to those planets and then treating the deviations as corrections to those basic motions. The concepts of relative, absolute, apparent, true, common and mathematical time were all present in the development of the project of constructing a time parameter appropriate for the purposes of mathematical astronomy, as that project existed at the time that Newton was writing, and therefore all three distinctions were empirically engaged at that time. These are distinctions that we can get at empirically, through the project of mathematical astronomy.

The intricacies concerning the treatment of time in mathematical astronomy were familier to Newton. In the scholium to the definitions he writes (Cohen and Whitman, 1999, p. 410):

In astronomy, absolute time is distinguished from relative time by the equation of common time.

In other words, we move from common time (a time adequate for the purposes of regulating our daily lives) to a time parameter appropriate to the needs of mathematical astronomy, and in so doing we move from relative time, on which common time is based, to absolute time, making use of the equation of common time and therefore of the mathematical properties of our time parameter. Newton further elaborates on the role of the equation of common time as follows (Cohen and Whitman, 1999, p. 410):

duration is rightly distinguished from its sensible measures and is gathered from them by means of an astronomical equation. Moreover, the need for using this equation in determining when phenomena occur is proved by experience with a pendulum clock and also by eclipses of the satellites of Jupiter.

The point he makes in this paragraph is that, in practice, we have strong theoretical reasons for believing that we have not yet found bodies (either celestial or terrestrial) whose periodic motions can serve as perfect clocks. The time parameter that would be measured by such perfect clocks, and that we 
have strong empirical reasons for adopting based on our investigations in astronomy and in terrestrial clock-making, is neither relative nor apparent. Thus, Newton knew very well that the "time" that is appropriate for astronomy, and thus the "time" that is appropriate for solving the problem of the system of the world, is mathematical, and is neither relative nor apparent. In specifying his three distinctions, he knew that they are distinctions that we can get our hands on empirically, and that we do this via the theories that we construct in order to account for the detailed motions of celestial bodies (the planets) and of terrestrial bodies (pendulum clocks). ${ }^{10}$

\section{Absolute, true and mathematical time in the project of the Principia}

We have seen that Newton had some empirical basis for making the distinctions between absolute and relative, true and apparent, and mathematical and common time (as I have interpreted that terminology), and that he had good empirical reasons for believing that the time parameter appropriate for mathematical astronomy is absolute, true and mathematical, rather than relative, apparent and common. I will now argue that each of these three conceptual distinctions suggested by mathematical astronomy is necessary for setting up the project of the Principia. This enables me to argue that the project of the Principia is a means for empirically investigating the characteristics of time that are associated with its being absolute or relative, true or apparent, and mathematical or common.

As noted above, the end goal of the Principia is to establish the "system of the world"; that is, to decide between the traditional geocentric, the Tychonic geocentric, and the heliocentric systems by establishing the true motions of the bodies in our planetary system. ${ }^{11}$ Successful completion of the project of the Principia thus requires true motion: without true motion, there is no determinate answer

\footnotetext{
${ }^{10}$ I have argued that Newton had good reason to believe that no material system is a perfect clock, and that time should therefore be considered absolute and not relative. My focus was on astronomical clocks, but Schliesser (2013) reminds us of the importance of seventeenth century advances in pendulum clocks, and their connections with time-keeping in astronomy. Newton was, of course, deeply immersed in this work too, especially through the tight interconnections between the study of the pendulum and of gravitation, and he was intimately engaged in studying the precise limitations and approximations involved in pendulum clocks. This work serves to reinforce the need for a distinction between the material clock and the absolute time that it approximates. See Schliesser's paper for much greater contextualization of Newton's treatment of time than I have given here.

${ }^{11}$ In the end, of course, Newton replaces all of these with a system in which none of these bodies remains at rest at the center of the system. Nevertheless, in doing so he establishes the true motions of these bodies.
} 
to the question of the system of the world. The project therefore requires the distinction between true and apparent motion.

Newton's proposed strategy for solving the problem of the system of the world was to systematically correlate forces with true motions. In the scholium to the definitions, Newton argues that true motion cannot be relative motion, and must therefore be absolute motion instead, by showing that on a relative conception of motion, true motions are not appropriately correlated with the presence/absence of forces. This is one purpose of the famous bucket experiment. ${ }^{12}$ Thus, insofar as true motion is necessary for the project, we also need absolute motion. But we cannot have absolute motion with relative time, since the resulting motion would then be dependent on the material bodies whose relative motions constitute relative time. Therefore, successful completion of the project of the Principia requires the distinction between absolute and relative time.

True motion also requires true time: there must be a unique time parameter proper to the system of the world, for if there is not, then a second "time" might give different conclusions concerning the motions and forces, and therefore concerning the system of the world. Thus, setting up the project of the Principia requires that we distinguish between true and apparent time.

If mathematical astronomy is the appropriate route for solving the system of the world, as Newton believed, then the need for distinguishing between mathematical time (with its metrical properties, and so forth) and common time is demonstrated by the need for an equation of common time. As noted above, Newton's proposed strategy for solving the problem of the system of the world was to systematically correlate forces with true motions, which in turn demanded a mathematical treatment of forces and motions as set out in Books I and II of the Principia. The specific mathematical properties required of the time parameter in these treatments stands in need for further investigation by Newton scholars, ${ }^{13}$ but it is immediately clear from the outset of the Principia that the time parameter must be metrical: Law 1 relies on equal intervals of time for the distinction between uniform and non-uniform motion, and this is at the heart of the distinction between the presence and absence of forces by which we are to arrive at the true motions.

The distinctions between absolute and relative, true and apparent, and mathematical and common time are therefore intimately tied to the project of the Principia. Newton had good reason to

\footnotetext{
${ }^{12}$ Cohen and Whitman, 1999, pp. 412-13. For discussion of the bucket experiment, see Huggett (2012) and references therein.

${ }^{13}$ Arthur, 1995; Palmerino, 2013.
} 
assert all three distinctions in setting up his project. The three distinctions are mutually independent of one another, and all three are needed for the purposes of his empirical project.

Newton also had good reason to believe from the outset that the time parameter appropriate for successful execution of his project would turn out to be absolute, true and mathematical, rather than relative, apparent or common. However, setting Newton's own position to one side, one cannot know at the outset whether the demands of the empirical project, as it unfolds, will indeed restrict the characteristics of the time parameter such that it turns out to be absolute, true and mathematical. This is something that can be settled only by pursuit of the project. I therefore disagree with Schliesser (2013) that only absolute (mathematical) time is connected to an empirically open question capable of being addressed by the methodology of the Principia. As we have seen, Schliesser claims that absolute time (in the sense of a time parameter for the solar system) is needed by Newton for his dynamics in order for Newton to "identify and assign accelerations to moving bodies in a consistent fashion", but that Newton's inclusion of something called "true time" (understood as an extension of absolute time from the "local temporal frame" of the solar system to spatial infinity) turns out to be a metaphysical commitment going beyond the demands of the Principia, rooted in Newton's rational theology. I think that Schliesser is right about the need for such a time parameter for the project of the Principia, and about its empirical status, but I think that each of the three distinctions (as I have interpreted the terminology) represent open empirical questions concerning the characteristics of the time parameter, and questions that are capable of being addressed by the methodology of the Principia.

The three distinctions are subject to empirical investigation in the first place through their connection to the problem of true motion. True motion is necessary for successful execution of the project of the Principia, but it is a contingent matter whether any such true motions exist: it might turn out that there are no true motions and thus there is no answer to the problem of the system of the world. Moreover, the project of the Principia enables us to probe the connections between true motion and time asserted above: for example, one might interpret Galilean relativity as indicating that true time is necessary but not sufficient for true motion, since there we have true time, and absolute (i.e. not relative) motion, but seem not to have true (i.e. unique) motion. This is an example of how the questions we are asking are transformed and become more fine-grained in the process of addressing them through the project of the Principia. There is much more to be said here concerning the execution of the project in the details of the Principia, in the uses of time that Newton makes in his mathematical arguments, his search for empirical clocks, and the interplay between these and his construction of absolute, true and mathematical time. It is by understanding these details, and their relationship to the 
empirical successes and failures of the Principia and later developments in physics, that we will find out the extent to which time turns out to be absolute and/or true and/or mathematical. The point that I want to stress here is that these aspects of the nature and structure of time are now tied to the details of empirical enquiry. All three questions of whether time is absolute or relative, true or apparent, and mathematical or common, have become empirically tractable.

\section{Empiricist metaphysics}

I have argued that in Newton's hands all three questions of whether time is absolute or relative, true or apparent, and mathematical or common, become empirically tractable through a project such as the Principia. But what is the significance of this conclusion for the metaphysics of time? Afterall, it is the demands of Newton's project that lead to the adoption of an absolute, true and mathematical time parameter: for the purposes of constructing a physics of matter in motion capable of addressing the question of geocentrism versus heliocentrism, it is absolute time rather than relative time (for example) that serves our needs. And it might be thought, therefore, that the results are restricted to the following form, "For the purposes of solving the system of the world, we must treat time as if it is absolute, true and mathematical", and that this claim leaves the deeper philosophical questions concerning the nature and structure of time untouched. But this would be a mistake.

To see why, we need to situate Newton's work in its philosophical context. Newton's Principia makes contributions to mathematics, mechanics, astronomy, physics and philosophy, and the text of the Principia should therefore be read in many ways. One of these is as a contribution to philosophy. I argue that we are right to incorporate Newton into the history of philosophy of time, not because his physics has implications for questions in philosophy of time which are to be drawn out by philosophers, but for his own direct contributions to philosophy of time through the conceptual distinctions that he makes and the methodology that he employs.

The most helpful philosophical context for these purposes is the work of Descartes. In Part II of his Principles of Philosophy, published in 1644, Descartes set out the framework of a project the goal of which is to provide an account of all the rich variety of the world as we experience it in terms of matter in motion, where that matter is characterized by a minimal set of properties (extension in Descartes's case), "motion" is restricted to local motion (i.e. motion from place to place), and the parts of matter move and interact according to specified laws. Since Descartes's laws are both spatial and temporal, space and time also fall under the scope of his project. It is this portion of Descartes's philosophy that 
Garber (1992) labelled "Descartes's metaphysical physics". It is indeed a "metaphysical physics" in the following sense: it is intended to give an account of the nature, structure, and operation of the material world. The concepts it employs, of matter, motion, laws and so forth, are not intended to be understood "as if" (i.e. serving an instrumental purpose relative to the project in hand), but as being metaphysically significant. To this metaphysical project, Descartes attached his rationalist epistemology of clear and distinct ideas.

Many of the important philosophical moves made by Newton are best understood as taking place within the framework of this project, and as responses to difficulties that Newton found in Descartes's execution of that project. They should therefore be read as contributions to the metaphysics of matter, motion, space and time, just as much as Descartes's. What makes this project look so different in Newton's hands is not the project itself, but the epistemology and methodology by which Newton sought to carry it out. Newton's methodology presses us to make the most of the actual world we find ourselves in as an epistemic resource in pursuing the metaphysical project set up by Descartes, and this fastidious attention to empirical details is an expression of Newton's empiricist epistemology. We are being offered an empiricist metaphysics, but a metaphysics no less. Thus, for the purposes of philosophy, it is important to read Newton's project as a continuation of Descartes's, and to assess it on these grounds.

As an aside, just to be clear: this is not the classical empiricism that gets discussed in the chapter after Newton in the Blackwell Companion (2013) mentioned in the Introduction to this paper. One of the problematic things that happens in early modern philosophy is that the common starting point for both rationalists and empiricists is our "ideas", which are cut free of any connection to the world and then the problem of knowledge becomes how to connect them back up again so that we can know anything about the world. But this is not a neutral starting point, of course, and it's not one which Newton accepts. He's an empiricist, in the sense that all our knowledge comes through our experience; but, for Newton, to know the world through our experiences is not to first know our "experiences" and to then know the world. He is clear that's a mistake, and he is clear about the methodological implications that follow.

Challenges to the significance of Newton's work for metaphysics should take place within this philosophical context. For example, one could challenge the value of Newton's contribution to metaphysics by rejecting the goal of Descartes's metaphysical physics (of giving an account of all the rich variety of the world as we experience it) as being irrelevant to the metaphysics of matter, motion, space and time. The level of detail at which Newton attends to the actual world could perhaps make this move 
tempting, but I think it would be a mistake. Unless we take the actual world as our guide, we risk constructing a metaphysics of matter, motion, space and time that fails to include the actual world among its possibilities. Newton's methodology requires us to take very seriously the goal of including the actual world among the possibilities we're addressing, and the metaphysical project set up by Descartes is therefore to be carried out with careful attention to empirical details.

One could also challenge the framework of the project (in terms of matter, motion and laws) or the methodology adopted for carrying it out. For those of an empiricist inclination, seeing the framework and the methodologies in action will be an important part of this assessment. In being allowed to prove themselves (or not) with respect to the goals of the project, they prove their utility (or not) with respect to the metaphysics of matter, motion, space and time.

If we adopt this approach, then the framework and the method will be judged by their results, and the significance of the project for metaphysics will depend in part on how fruitful the project proves to be with respect to the goal of giving an account of all the rich variety of the world as we experience it. Failure to succeed with respect to the goal is a legitimate route by which to challenge claims about the significance for metaphysics of the conceptual investigations carried out within the project. Notice that this depends on assessing the detailed empirical success of the project, and this is characteristic of the empiricist metaphysics that I am describing in this paper.

My claim is that Newton's Principia makes direct contributions to the metaphysics of space, time, matter and motion, and that this becomes visible if we read the Principia as a contribution to philosophy, in its philosophical context, by beginning with our metaphysical questions concerning space, time, matter and motion as they were prior to Newton, and seeing what happens to them through the process of Newton's attempts to carry out Descartes's metaphysical physics project. In particular, Newton's results concerning time are not of the above "as if" restricted form, but are contributions to our investigations into the nature and structure of time, and can be read as offering an empiricist metaphysics. To see how we address general questions about the nature of time by means of such an empiricist metaphysics, we return to Newton's absolute, true and mathematical time. For each of the three distinctions that Newton makes, there are issues concerning the nature and structure of time that we're being asked to address, and for each of these Newton is providing empirical purchase such that, as we shall see, addressing these questions is no longer a matter of general philosophical reasoning, but depends on the details of empirical enquiry. For those of us who value history of philosophy as a route towards understanding the philosophical questions that we have today, we know how important it is that we tell and re-tell that history; the case of Newton on time is one example of how we also need to 
tell and re-tell the history of physics, read as a contribution to philosophy, in order to re-tell the history of philosophy.

\section{Time for empiricist metaphysics}

\subsection{Absolute time}

The question of whether time is absolute or relative concerns the relationship between time and change. The general question is whether time is an aspect of material change, or somehow independent of matter. In a world such as Descartes's, where all change is accounted for in terms of local motion, the question is whether time is an aspect of the relative motions of bodies, or somehow distinct from these. Sharpening the question a little, this becomes: are there any actual relative motions that can serve as clocks for the material goings-on in the world, such that our project (of providing an account of all the rich variety of the world as we experience it) is tractable? Newton's answer is, of course, no; according to Newton, time is absolute, not relative.

My point here is not Newton's answer, but the method by which this question is to be addressed. The project of the Principia demands the conceptual distinction between absolute and relative time, because (if all bodies are perhaps interacting by means of forces, as yet unknown) there is no material system to which we can turn a priori as a clock. It is then an empirical matter whether there is in fact any material system that acts as a perfect clock relative to the absolute time parameter of the project. Thus, in Newton's hands, the question of whether time is relative or absolute has been turned into an empirically tractable question, and one whose answer depends on the details of empirical enquiry; the appropriate arguments for deciding the question are no longer those based on broadly $a$ priori considerations.

A brief comparison with Aristotle may perhaps be helpful. For Aristotle, time is an aspect of material change, and the motion of the outermost heavenly sphere is the change by which all other changes are measured. In his view, it is not possible to discover empirically that the motion of the heavens (or, equivalently kinematically, the rotation of the Earth) is not uniform and regular. Newton's method leaves the question open. It begins from an assumption of at least approximate uniformity in this rotation, and then harnesses the details in the phenomena to construct a time parameter and to examine the speed of the Earth's rotation with respect to this parameter. As it turns out, it's very hard to tell that the rotation of the Earth (i.e. sidereal time) isn't perfectly uniform, i.e. that it's not a perfect 
clock relative to Newton's time parameter. It took until 1927 to show beyond doubt that there are tiny irregularities in the rotation of the Earth. But again, it is the mode of argument rather than the conclusion that I want to emphasize. We're not arguing from general principles, or from experience in general, in order to establish our conclusion; rather, we are having to pay attention to the minute details of empirical enquiry in order to address the question of whether there are actual material changes of which time is an aspect, so that these material changes constitute a perfect clock, or whether there are no perfect clocks so that time itself is distinct from material change. It is in this sense that we are doing empiricist metaphysics.

Gassendi's discussion of place, space and time (in The Syntagma, see Gassendi, 1972, pp, 383398) provides another helpful contrast. Newton's treatment bears strong resemblances to Gassendi's discussion, conceptually and in its phrasing (see McGuire, 1966). However, two striking differences are pertinent to our purposes. First, Gassendi treats place and space first, and proposes that we can learn about time by drawing on analogies with space. Second, the arguments Gassendi gives are of the general kind that I am emphasizing are not found in Newton. He argues in general conceptual terms for why time must be prior to and distinct from the motions of material things. It is a methodology of argumentation that Aristotle would have recognized. Gorham (2012) situates Newton's philosophy of time within this tradition of metaphysical arguments for and against the possibility of empty space, in which conclusions about time are drawn by analogy with conclusions about space. Gorham points out that Newton does not argue for absolute time using the kind of metaphysical arguments traditionally offered in support of absolute space. I think that the absence of traditional metaphysical arguments for absolute time in Newton's philosophy of time is because the kind of argument he is offering is different. Newton is offering a methodology in which these questions become empirically tractable, so that a different type of argumentation is required. And I think this represents a big change in philosophy of time. In the wake of Newton's Principia, those who accept Descartes's project (as characterized above) have no choice but to develop their philosophy of time, and their account of the relationship between time and material change, in a way which takes into account the types of argument developed by Newton rather than only those that proceed by general conceptual argumentation.

One final comment about the conclusion that Newton draws, i.e. that time is absolute. Newton's type of argument does not prove that there are no relative motions that constitute a perfect clock, of course. Rather, his argument relies on a conceptual distinction between absolute and relative time, and then provides an empirical argument for the claim that in fact time turns out to be distinct from relative motions of material bodies. And therefore, as such, it is open to empirical refutation, by the finding of a 
material system that constitutes a perfect clock. ${ }^{14}$ But a good empiricist metaphysics proceeds on the basis of empirical evidence and remains open to revision on the basis of future empirical findings, so this is no objection to the claim that, so far as we have been able to ascertain, time is not relative and is distinct from material change.

\subsection{True time}

Apparent time yields multiple times: apparent solar time, apparent lunar time, and so forth, ticking irregularly with respect to one another. Among the reasons that Gassendi gives against a relationist conception of time is that such an identification of time with actual motions would lead to multiple times. Gassendi asserts that this is to be rejected in favor of a single, unifying time (see Gassendi, 1972, pp. 393-4). In Gassendi, the question of whether there is one time or many is to be settled by broadly $a$ priori argumentation, whereas in Newton's hands the issue becomes empirically tractable.

Newton distinguishes between true and apparent time. True time, just like true motion, is a property of the body or system itself, not of the appearances. In the equation of common time, we search for a single time parameter to be used in common for all bodies, relative to which we can construct a theory of their motions. If successful, this time parameter corresponds to the true time of the system of the world. For example, the lunar day and the solar day are different apparent measures of time, ticking irregularly with respect to one another, but belief in true time is the belief that (i) underlying these differing apparent measures is the one true time which these motions approximate to a greater or lesser extent, and crucially (ii) the irregularities in the motions of the moon and the sun relative to true time can be precisely quantitatively accounted for, without remainder, by our theory of forces by which the bodies in our system interact. The assertion that time is true is the assertion that there is one time, not many, in this specific sense.

True time for Newton, I suggested, is time that is unique and proper to the system of the world. The "system of the world" for Newton is the solar system, but the issue of true time (on the view I advocate) is system-relative and therefore independent of whether the "system of the world" is our solar system or some other system or the material universe as a whole. The issue is whether, for the system of interest, there is any such thing as true time. In the context of Descartes's project, and in the

\footnotetext{
${ }^{14}$ Moreover, the finding of such a clock would generate further questions about the relationship between that clock and material sub-systems of the universe such that the ticking of the perfect clock could be constitutive of time for these sub-systems.
} 
hands of Newton, this is transformed into the following empirical question: can we construct a satisfactory physical theory for this system using a single time parameter? ${ }^{15}$

There are at least two ways in which this could fail. One possibility is that we could get close but not quite there, so that we are left with irregularities that cannot be accounted for either by modifying the time parameter or in terms of forces: we are not able to come up with a satisfactory physical theory using a single time parameter, and so we are unable to supply this empirical warrant for the existence of a true time associated with that system. A second possibility concerns a specific way in which this might happen: subsystems dominated by different forces might tick irregularly with respect to one another with no common underlying metric. For example, there is no guarantee a priori that an atomic clock, governed primarily by the laws of quantum mechanics, will tick regularly with respect to a pendulum, whose rate of ticking is governed primarily by gravitation; the two could turn out to tick irregularly with respect to one another with no common underlying metric. There would then be no such thing as "true time" for any system in which both quantum mechanics and gravitation play a role. Specifically, were it to happen for the solar system, then there would be no such thing as true time for the system of the world.

It is, therefore, an empirical question - and an open empirical question at that - whether any such single time parameter can be constructed. In Newton's hands, the appropriate methodology for tackling the question of whether there is one time or many has been utterly transformed. Whether there is one time or many is something that we discover not through a priori argumentation alone but through attention to the details of empirical enquiry. The question of whether there is one time or many has become an empirically tractable question, one which is appropriately addressed by paying attention to the details of empirical enquiry, and through the progress of that enquiry.

\subsection{Mathematical time}

Newton's third distinction between mathematical and common time brings us back to the relationship between time and change. Newton's assertion that time is mathematical includes the claim that it is metrical. What this means is that time is a measure of change: changes occur regularly or irregularly, and faster or slower, with respect to the metric of time, and the comparative length of time between pairs of events is determined by this metric structure. (This is independent of whether time is absolute

\footnotetext{
${ }^{15}$ Thus, the question also becomes more complex: for any given system, we can ask whether time is true, and also for the collection of all systems we can ask whether time is true.
} 
or relative, and so of whether there are any material clocks, or not. It is also independent of whether time is true or apparent.) As we have seen, time without this much structure seems to be insufficient for the needs of the project of the Principia. Thus, pursuit of the project of the Principia is in part an investigation of the claim that the time parameter of the Principia must be metrical, and in this way the question of whether time is mathematical has been transformed into an empirical question. Indeed, just how much structure the time parameter must have, in order to fulfill the needs of Descartes's project, is an open question to be explored through the process of empirical enquiry, and it is one which remains an open question today.

There are some interesting conceptual intricacies that arise in the wake of the assertion that time is metrical, and I will finish by offering some comments on these. Following the assertion that time is metrical, there remains the possibility of a gap between time as a measure of change, in and of itself, and time in relationship to actual material changes taking place in the world: why think that the two tick in harmony? Relationists close the gap by identifying units of time with the rhythm of repeating material phenomena, but if time is absolute, as Newton believed, then it is not clear why the ticking of clocks should tell us anything about the metric of time. While time is a measure of change, we measure time by means of material change, and if the metric of time were capricious in relation to material change then material clocks would not measure time. This is not as silly as it sounds: Newton was very alert to the question of whether a unit tick of a clock at one time measures a unit of duration at another time; it is a live possibility for Newton that the length of time between any two ticks of a clock may not be equal. In order to see the significance of this more clearly, it is instructive to consider the difference between Newton's treatment of spatial interval and his treatment of duration.

Spatial intervals are measured by rulers; rulers are bodies, and according to Newton place is a part of space which body fills. A body of unit length by definition fills a region of space of unit length. There is therefore no distinction between the metrical characteristics of bodies, as occupiers of space, and the metrical characteristics of the parts of space that they occupy. All rulers are, in this sense, perfect rulers: no question arises as to whether a unit ruler measures a unit interval of space, and indeed whether that unit ruler at one location and at one time measures a unit interval of space when moved to another spatial location and/or at another time.

By contrast, not all clocks (indeed perhaps no clocks) are in an analogous sense perfect clocks. And this is the point about the possible capriciousness of time: a unit tick of a clock at one time may not measure a unit of duration at another time. For Newton, there is a gap between duration and our 
measure of duration that does not arise for length and our measure of length. This is why Newton need not specify explicitly that space is mathematical, whereas he must - and does - do so for time. ${ }^{16}$

The gap between duration and our measure of duration therefore presents us with an epistemic problem, in that we cannot measure duration directly, but only indirectly by means of motion. Closing this gap involves two steps. First, we have to specify that time is metrical, and this is something Newton does. Then, we have to establish a relationship between this metric of time and the "ticking" of material clocks, so that our means of measuring duration is not utterly unreliable as a guide to the metric of time. Newton does this by stipulating that time "flows equably": the metric of time is not capricious in relation to physical processes. ${ }^{17}$ To say that time "flows equably" is to say not only that it is a measure of change, but that the processes and changes that we experience as regular are regular, at least approximately, with respect to time. Were it otherwise, then the metric of time would be epistemically utterly inaccessible to us.

Thusfar, Newton has been making clear what demands are placed on our concept of time by the requirement that material clocks enable us to measure intervals of absolute time. The evidence that time flows equably is the practical success achieved by astronomers with the equation of time, and by Huygens with the pendulum clock. There may be no perfectly equable motions to be found in the material world, since there may be no material clock that ticks precisely in accordance with the metric of time, but we have good empirical reasons in support of the claim that absolute and true time flows equably.

I think that the issues here are more complex than those raised by absolute and true time, and that the subsequent history of work on space and time bears this out. The relationship between material processes and space and time continues to be rich philosophical territory in foundations of physics, in which a wide array of positions is available. There are disputes over the priority of space and time versus matter, over the minimal structure that we must attribute to space and time, over the nature of the relationship between spacetime and matter, and of how it is that rods and clocks, such complex material systems, overcome that complexity to somehow tell us something about the structure of the spacetime in which they are situated and move. It is not at all obvious how to resolve these questions empirically, and what this makes vivid is the sophisticated reasoning that is involved in bringing the empirical to bear

\footnotetext{
${ }^{16}$ Moreover, this marks a difference as compared to mass, as a measure of matter, and momentum, as a meaure of motion, for example, where Newton is able to formulate quantities that do not suffer from the same kind of epistemological gap. In the case of time, it seems that no amount of conceptual work will remove the gap.

${ }^{17}$ Arthur (1995) argues, though for different reasons, that we should regard Newton's assertion that time is mathematical as asserting that it flows equably, as well as that it is metrical.
} 
on such difficult philosophical questions. Nevertheless, what is also clear is that it is through the process of this very engagement with the details of empirical enquiry that progress on these questions is to be made. Again, the methodological point is the one that I wish to emphasize, not the answer to the question of whether or not time is mathematical. It is because of the moves that Newton makes that we uncover all the philosophical complexity associated with this claim in the first place.

\section{Conclusions}

I have argued that Newton is very deliberate when he sets out his three distinctions between absolute and relative, true and apparent, and mathematical and common time: each distinction is different, and each is needed for setting up the project of the Principia. I have also argued that all three distinctions mark open empirical questions in the context of the Principia (though I make no claim that this was Newton's own view). The questions of whether time is absolute or relative, true or apparent, and mathematical or common become empirically tractable in the context of the project of the Principia (or some such project). Moreover, the significance of this conclusion is not local to the Principia. If we read the Principia as making a contribution to Descartes's "metaphysical physics", then we can read the moves that Newton is making as contributions to that metaphysical project, albeit via a very different epistemology and methodology. Read in this light, we see that Newton's distinctions are connected to questions concerning the nature and structure of time. Newton transformed the methodology by which these questions should be addressed, providing empirical purchase on them and rendering them empirically tractable. Prior to Newton, such questions as whether time is relative (depending on the actual motions of bodies) or absolute (independent of these motions), and as whether there is one time or many, were questions that could be tackled independently of the details of empirical enquiry. In the wake of the Principia, this is no longer the case: any legitimate exploration of these questions must take into account the kind of detailed empirical enquiry pursued in the Principia. Philosophical progress has been made.

In addition to these specific conclusions concerning an appropriately empiricist metaphysics of time, there is a more general methodological message that is perhaps worth highlighting. Disputes in philosophy of time over how to understand the significance of special relativity, for example, are hampered by difficulties over how to read the developments in physics as contributions to philosophy. Disputes arise as to whether the philosophical challenges posed by relativity of simultaneity in special relativity can be safely ignored by philosophers since special relativity is a false theory, and over 
whether, even if special relativity is to be taken into account, the dispensability of a privileged present in special relativity implies anything about the existence or otherwise of a privileged present, metaphysically. ${ }^{18}$ These disputes seem to me poorly framed, because they fail to read special relativity itself as arising through a diachronic process of philosophical engagement with our everyday concepts of space and time, a process which has clarified and transformed those concepts (see DiSalle, 2006). The example I have discussed in this paper is Newton's work on duration, and I have said nothing about simultaneity, which is more of a hot topic in contemporary metaphysics. But similar work to that which I have done here can be done for simultaneity. The question of whether time flows is another topic that has received attention recently and, as my remarks on mathematical time above indicate, I think that attention to the role of this claim in Newton's project help clarify how such a claim should be understood. By reading developments in physics as a part of the history of philosophy, we can make visible the ways in which physics contributes to the conceptual clarifications and transformations of the very questions that we are asking about time, and the means by which aspects of our oldest questions concerning time are rendered empirically tractable. ${ }^{19}$

The Oxford Handbook of Philosophy of Time (2011) and the Blackwell Companion to the Philosophy of Time (2013) both contain sections written by metaphysicians (on such topics as presentism, persistence, fatalism, and tense) and sections written by philosophers of physics (on time in classical and relativistic physics, and in cosmology and quantum gravity). Though I have little empirical evidence to offer you, I am doubtful how much each group of philosophers reads one another's chapters: if cross-referencing is anything to go by, they are not really talking to one another, and one reason is their differing methodologies. A more historically driven methodology, in which both philosophy and physics are read diachronically as contributions to our ongoing philosophical conversation, would enable both parties to speak to one another in more fruitful ways.

\section{Acknowledgements}

I am grateful to the participants in my graduate spacetime seminar (spring 2013), and to our visiting speaker Eric Schliesser. My thanks to the Pittsburgh Center for Philosophy of Science, the Stanford Philosophy Department, and the London School of Economics, and to all those present at my talks.

\footnotetext{
${ }^{18}$ For a recent example where these issues arise, see Zimmerman, 2013.

${ }^{19}$ See, for example, Smeenk, 2013, who discusses the transformation of old questions by developments in cosmology.
} 
Special thanks to Ori Belkind, Meghan Dupree, Jennan Ismael, Anja Jauernig, Xavi Lanao, Tom Ryckman, George Smith, Monica Solomon, Michael Strevens and Zanja Yudell for their comments and discussion.

\section{References}

Arthur, R. T. W., 1995. "Newton's Fluxions and Equably Flowing Time". Studies in History and Philosophy of Science, 26(2), pp. 323-351.

Audoin, C., and Guinot, B., 2001. The Measurement of Time. Cambridge: Cambridge University Press.

Cohen, I. B., and Whitman, A., 1999. Isaac Newton, The Principia, Mathematical Principles of Natural Philosophy: A New Translation. Los Angeles: University of California Press.

Descartes, R., 1991. Principles of Philosophy. V. R. Miller and R. P Miller, trans. Dordrecht, Boston, London: Kluwer.

DiSalle, R., 2006. Understanding Space-Time. Cambridge: Cambridge University Press.

Gassendi, P., 1972. The selected works of Pierre Gassendi. C. B. Brush (ed. and trans.). New York, London: Johnson Reprint Corporation.

Gorham, G. 2012. "The Twin-Brother of Space': Spatial Analogy in the Emergence of Absolute Time". Intellectual History Review, 22(1), pp. 23-39.

Hall, A. R., and Hall, M. B., 1962. Unpublished Scientific Papers of Isaac Newton. Cambridge: Cambridge University Press.

Huggett, N., 2012. “What did Newton mean by “Absolute Motion'?”. In Janiak and Schliesser (2012), pp. 196-218.

Newton, I., 2004. Philosophical Writings. A. Janiak (ed.). Cambridge: Cambridge University Press.

Janiak, A., and Schliesser, E., 2012. (Eds.) Interpreting Newton: Critical Essays. Cambridge, New York: Cambridge University Press.

McGuire, J. E., 1966. Body and void in Newton's De Mundi Systemate: some new sources. Archive for the History of Exact Sciences 3, 206-248.

Palmerino, C. R., 2013. "The composition of space, time and matter according to Isaac Newton and John Keill". In S. Roux (ed.), The Mechanization of Natural Philosophy (Boston Studies in the Philosophy of Science). Springer. pp. 117-142.

Schliesser, E., 2013. "Newton's philosophy of time". In H. Dyke and A. Bardon (eds.), A Companion to the Philosophy of Time, First Edition. Edited by Heather Dyke and Adrian Bardon. John Wiley and Sons, Inc., pp. 87-101. 
Stein, H., 1970, "Newtonian Space-Time”, in R. Palter (ed.) 1666: The Annus Mirabilis of Sir Isaac Newton. Cambridge, Massachusetts; London, England: MIT Press. pp. 258-284. 